\title{
J'ai oublié
}

\section{Olga Duhamel}

If only I could remember the name of that bar on the edge of the beach. Wildly we danced on its unlit floor, as the tequillas were mixed at the bar. Later, during the winter, I forgot the heat wave that lasted months, scorching everything. I forgot the mediocre contentment with the difference between the sexes. I also forgot the joy of discovering your difference. The sense of forgetting in Olga Duhamel's text subtly shifts between loss of memory, confused recollection, the act of changing one's mind, and the refusal of a certain conceptual ordering of the world. Speaking from a position which has forgotten "how exactly the sexual orientations are divided," the narrator addresses a similarly unstable, multiple other, recollected through a chain of associations which moves through masculine and feminine, second and third-person pronouns: "I think of you now, night and day. It is difficult to explain. I think of him, of D. And with him, I think of S, of her beauty."

Si je me souvenais du nom de ce bar. Si j'arrivais à me souvenir du nom de ce bar où il y avait une toute petite piste de danse, sans éclairage. On dansait comme des déchaînés sur cette piste de danse, avec les tequilas frappées au bar. Comment ça s'appelait?

Les gens disaient : C'est de la New beat et on redansait là-dessus. Il me semble que c'était toujours la même chanson : She'd rock to the beat, she'd rock to the beat. C'était dans un endroit reculé dont j'arrive pas à me rappeler le nom.

On frappe les tequilas-schweppes sur le bar. Et puis on danse sur cette même chanson.

En sortant de cet endroit, on va sur la plage. Près de la frontière espagnole, on avait fait ça aussi. Rester toute la nuit dans une boîte médiocre je ne me rappelle que de ces bouteilles vertes de Heineken. Et je parle à des gens que je ne connais pas. Entre la piste de danse et le bar. Les autres sont sortis avant, et je vais les rejoindre sur la plage.

Nager, dormir, en attendant l'ouverture des premiers cafés du bord de mer, pour boire des cafés. 
Mais il ne faut pas oublier la chaleur qu'il fait toujours. Après, l'hiver, je ne peux plus me souvenir de cette canicule qui dure des mois - qui assèche tout.

J'ai oublié.

La médiocrité de l'infini contentement de la différence des sexes. J'ai oublié, la joie sans cesse renouvelée de te trouver différent.

La fascination de l'évidence, la pulsation - parce qu'on serait fait l'un pour l'autre - des corps imbriqués.

J'ai oublié aussi le plaisir de dépasser l'évidence. La communauté des filles. J'ai oublié.

Mais te tenir tout contre moi.

J'avais oublié comment se divisaient exactement les orientations sexuelles. Ainsi, beaucoup de gens disaient que les hommes et les femmes ensemble produisaient des enfants, que c'était comme ça. Ah bon. Ah d'accord.

J'essayais de mémoriser la vérité et j'oubliais à mesure.

Des pilules, des préservatifs, des éponges remplies de spermicides, des stérilets; tout ça pour servir d'aide-mémoire. Des noeuds dans le mouchoir.

Pas facile de se rappeler. Quand ça veut pas se graver dans le cerveau. Il suffit de vouloir se rappeler d'un truc pour l'oublier. Alors qu'on fredonne toute la journée un air vague entendu par hasard le matin.

J'oublie sans le faire exprès.

J'ai oublié ce flirt perpétuel avec les hommes, rouler des hanches, vouloir te séduire. Hommes et femmes avec vos sourires curieux. Je me souviens juste d'être aspirée par toi.

Dans des boîtes de nuit avec une enseigne qui clignote. Dans un bar louche - gla gla gla. Dans mon appart, quand on a dansé. On était hystériques. On chantait trois chansons différentes, en même temps.

Dans les grands cafés élégants, j'ai oublié.

Oublié de te dire quelque chose d'important au fond. Je t'aime. Ce genre de refrain.

Sous le mot «toi», il y a eu une série de visages qui ont défilé et qui chaque fois, ressemblaient - c'était frappant - à toi!

Je me suis juste souvenue de ces journées au lit. Boire du champagne, des vins fins, des cafés forts au lit avec toi.

Te prendre par les hanches. Ensuite arpenter les rues à la recherche de cigarettes et de quelque chose à manger. 
Nappes de plastique sur les tables, bougies au bout de leurs mèches, des plats de cacahuètes, du punch rouge. Et des verres partout et la samba qui se joue dans le noir à peu près. Tout ça dehors. Parce que j'ai oublié.

Cette multiplicité soudaine, parce que j'ai oublié.

Bord de mer. Et tout d'un coup je me sentais si jeune, ma mère aussi était jeune et même ma chienne qui se jetait de front dans les vagues était jeune. Jeune était le mot pour bonheur et l'âge n'avait plus d'importance. Chardons bleus, plantes grasses, on se laissait chauffer par le soleil.

Disco. Trois buvettes brûlées par le soleil. Et rien ne pousse sur les montagnes. Que des petits trucs desséchés. C'est superbe. Les gens boivent beaucoup à cause de la chaleur bien sûr. Même avec le vent de la mer, il fait très chaud. Des gens se cachent de la chaleur dans les buvettes. Les chiens ne remuent pas.

Dans les endroits avec de l'ombre, ça bourdonne les insectes. Sous le soleil, rien.

Changer d'avis. J'ai changé d'avis.

Sur la plage de galets, quelques hommes fument des cigarettes qu'ils enfoncent à la fin sous les galets. Les cigarettes, même sous le soleil, ne leur donnent pas la nausée. Ils sont frileux, mais se jettent quand même à l'eau d'un coup.

C'est à toi que je pense maintenant. Tous les jours, la nuit. Je ne peux pas expliquer très bien. C'est à lui que je pense, à $\mathrm{D}$. Et c'est avec lui que je pense à S., à la beauté de S. Elle me prend par les épaules, elle est distraite. S. a sa main sur mes épaules. Elle est très triste dans la soirée. Pourtant des gens ont accroché des lumières colorées en guirlande. Il y a la musique, les gens se regardent et dansent. Elle aussi danse, avec des hommes qui viennent la chercher. Je regarde les pas. Je ne sais pas comment dire, tous ses gestes m'émeuvent. Elle fait comme si elle s'amusait quand un homme lui propose de danser. Tout est désir ce soir chez S., sans qu'elle y pense. Je sais que tu serais d'accord, que tu la trouverais belle aussi. Elle passe sa main sur mes épaules et on traverse la foule, les éclats de voix, la fumée des grils à merguez, les verres de plastique. Elle pense à autre chose et moi je la suis, tout contre elle.

Fontaines d'eau non-potable. Canicule trop forte pour fumer des cigarettes. Pas assez d'air. Cette nuit il fera peut-être miraculeusement frais.

Lui toucher l'épaule, même si je ne le connais pas, pour le réconforter, pour m'excuser de lui avoir dit que l'eau était empoisonnée. Un homme 
boit l'eau de la fontaine.

Il fait trop chaud pour boire du vin. Trop chaud pour le chien.

Un enfant est mort $d$ 'hydrocution.

On distribue des bouteilles d'eau aux chauffeurs d'autobus.

J'ai oublié. Des femmes m'ont parlé du malheur des homosexuels. J'y avais pas pensé, mais ça m'a fait penser au malheur hétéro, à une spécificité de ce malheur. J'ai chassé cette idée de ma tête : tout ça n'avait pas de sens. Tout ça, il a fallu que j'oublie. Oublier votre organisation du monde. Ça fait un peu prétentieux dit comme ça, mais en vérité c'est simple. Être têtue comme une mule. Faire à sa tête, aller tête première. Après, j'ai oublié. Et j'ai peine à me rappeler aujourd'hui. Juste je sais $q^{\prime}{ }^{\prime} i l$ faut bien choisir ce dont on se rappelle. Chasse les conneries de tes pensées pour aller libre.

Malheur des pauvres, malheur des bêtes, malheur des aveugles, malheur des gros, malheur des maigres, malheur des stars qui se suicident aussi. Malheur des femmes, malheur des hommes ravagés aussi. Du malheur comme concept, il n'y a rien à tirer pour la lutte. Comprendre de quoi parle cette vue de l'esprit qu'on appelle universalité, c'est peut-être la seule chose à laquelle peut servir intellectuellement le malheur.

L'aquarium a été sale un moment. Le dernier poisson est mort mais à présent, c'est différent. L'eau boueuse, tout ça s'est transformé. Les couleurs de l'aquarium sont redevenues chatoyantes, même plus encore qu'elles ne l'étaient auparavent. Enfin, je ne sais plus exactement mais à l'intérieur de l'aquarium aujourd'hui, toute la piscine que l'on voyait tout en bas, toute l'eau de la piscine, turquoise, avec de la vapeur, les lampes sous l'eau, la transparence bleutée - se retrouve dans l'aquarium ce soir. Le poisson rond et argenté revit. Et autour de lui il y a plusieurs petits poissons argentés qui nagent. Et je n'avais pas vu, mais il y a d'autres poissons avec des petits poissons eux aussi, des poissons mauves, très lumineux et des poissons jaunes et des poissons vraiment bleus. Un grand aquarium tout d'un coup, avec des feuilles et des tiges d'algues en suspension et des roches colorées au fond.

À un moment, j’ai rencontré un homme qui m'a dit : Fais pour que tu sois heureuse. Et j'ai tout bien écouté. Toi aussi tu m'as dit : Fais pour que tu sois heureuse. Je t'ai écoutée, je crois. Le mieux que j'ai pu.

Et le reste, j'ai oublié.

Parfums très sucrés qui enveloppent l'air. Après avoir passé quelque temps à pleurer, ce n'est plus la peine de pleurer. Toute la mesquinerie du monde s'évanouit dans le parfum des fleurs. Un groupe peut bien jouer de la musique dans un bar ordinaire, le plus fort possible, je ne sais pas, cette odeur de fleurs me frappe plus. 